excretion, only small differences subsist between fat except for stearic acid showing the following values (p. Ioo) : I, 72 ; II, 57 ; III, 72 ; IV, 3I. The energy and nitrogen digestibility and the nitrogen retention coefficient of the different diets were the following : I, 84-85-32; II, 87-88-36 ;III, 84-86-33 ; IV, 86-87-29 (significant differences in group II). As for calcium and phosphore digestibility, no difference is found between the diets. The metabolizable energy values of the different fats studied are the following : coconut 8873 ; tallow 7722 ; maize oil 8356 (calories/g).

\title{
EFFETS DE L'INTRODUCTION DE SON DE BLE DANS UN RÉGIME A BASE D'ORGE CHEZ LE PORC EN CROISSANCE-FINITION, EN PRESENCE OU NON DE LITIÈRE
}

\author{
Y. HENRY, D. BOURDON et M. CHAMBOLLE \\ avec la collaboration technique de B. DABIEL \\ Station de Recherches sur l'Elevage des Porcs, \\ Centre national de Recherches zootechniques, 78 -Jouy-en-Josas \\ Institut national de la Recherche agronomigue
}

\section{RÉSUMÉ}

En relation avec l'extension des techniques modernes d'engraissement du Porc èn confinement, deux expériences ont été réalisées sur porcs en croissance-finition, afin d'étudier les effets de l'introduction d'un aliment cellulosique (son de blé), dans une ration à base d'orge et de tourteau de soja, sur les performances de croissance et la composition corporelle, en présence ou non de litière. La réduction de la valeur énergétique du régime de 3 roo à 2 goo kcal d'énergie digestible par kg, par incorporation de 15 p. Ioo de son de blé, chez le Porc nourri selon son appétit, se traduit principalement par une augmentation de l'indice de consommation ( 7 p. Ioo), accompagnée d'une légère dépression de la croissance en présence de paille (590 g/j contre 62I, entre 30 et $90 \mathrm{~kg}$ de poids vif), mais seulement pendant la phase de finition. Par ailleurs, l'introduction de ro $p$. Ioo de son dans le même régime à base d'orge, correspondant à un abaissement de la valeur énergétique à $3000 \mathrm{kcal}$ d'énergie digestible par kg, en l'absence de litière, permet d'obtenir des carcasses plus maigres, sans qu'il en résulte une détérioration de l'indice de consommation. 


\title{
SUMMARY
}

\section{EFFECTS OF WHEAT BRAN IN A BARLEY-SOYBEAN OIL, MEAL DIE'T \\ ON THE PERFORMANCES OF THE GROWING-FINISHING PIG, WITH OR WITHOUT S'TRAWBEDDING}

Following the development of recent techniques of fattening pigs in confinement, two experiments were conducted on growing-finishing pigs in order to study the effects on srowth performance and carcass characteristics (with or without strawbedding), of including fiber (wheat bran) in a barley oil meal diet. Including I 5 p. Ioo wheat bran to decrease the energy value of the diet from 3 roo to 2900 digestible $\mathrm{kcal}$ per $\mathrm{kg}$ for full hand-fed pigs, leads to an increase of the feed conversion ration ( $+7 \mathrm{p}$. IOO). This is accompanied by a slight growth depression for pigs raised on straw ( $590 \mathrm{~g} /$ day vs $62 \mathrm{I}$ between 30 and $90 \mathrm{~kg}$ bodyweight), but only during the finishing phase of growth. On the other hand, when we introduce Io p. Ioo wheat bran in the same basic diet (corresponding to an energy value decrease down to 3 ooo digestible kcal per kg, without strawbedding) leaner carcasses are obtained without any deleterious effect on the feed convertion ration.

\section{UTILISATION DES DÉCHETS DE BANANE DANS L'ALIMENTATION DU PORC EN CROISSANCE}

\author{
J. LE DIVIDICH et I. CANOPE \\ Station expérimentale de Zootechnie, \\ Centre de Recherches agronomiques des Antilles-Guyane, Petit-Bourg \\ Institut national de la Recherche agronomique
}

\section{RÉSUMÉ}

Les déchets de banane constituent un produit riche en eau et en minéraux, mais très pauvre en azote. La présente étude nous a permis de montrer qu'ils peuvent constituer en pays tropical (Guadeloupe) un aliment pour le Porc en croissance et peuvent représenter jusqu'à 55 p. Ioo de la matière sèche ingérée. Le broyage et surtout la cuisson améliorent sa valeur alimentaire et même sa digestibilité. La digestibilité de la matière organique de la banane déterminée par différence varie de 83 à $87 \mathrm{p}$. Ioo pour le fruit cuit, celle de l'énergie variant de 75 à 82 p. roo. Par 